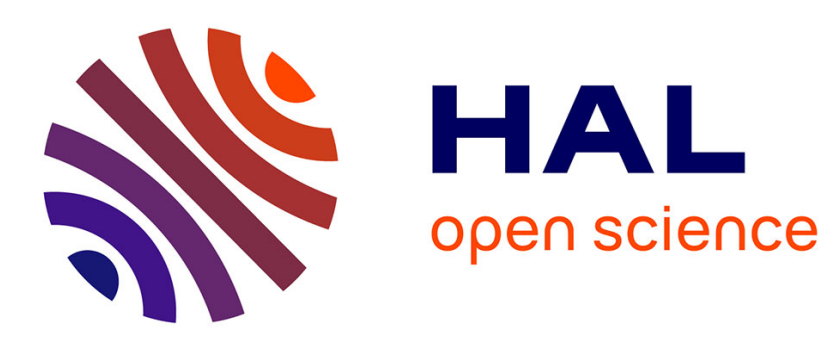

\title{
Les petits espaces insulaires face à la variabilité de leur insularité et de leur statut politique
}

François Taglioni

\section{To cite this version:}

François Taglioni. Les petits espaces insulaires face à la variabilité de leur insularité et de leur statut politique. Annales de géographie, 2006, 652, pp.664-687. halshs-00172396

\section{HAL Id: halshs-00172396 \\ https://shs.hal.science/halshs-00172396}

Submitted on 16 Apr 2017

HAL is a multi-disciplinary open access archive for the deposit and dissemination of scientific research documents, whether they are published or not. The documents may come from teaching and research institutions in France or abroad, or from public or private research centers.
L'archive ouverte pluridisciplinaire HAL, est destinée au dépôt et à la diffusion de documents scientifiques de niveau recherche, publiés ou non, émanant des établissements d'enseignement et de recherche français ou étrangers, des laboratoires publics ou privés. 


\section{LES PETITS ESPACES INSULAIRES FACE À LA VARIABILITÉ DE LEUR INSULARITÉ ET DE LEUR STATUT POLITIQUE}

François Taglioni

Armand Colin / Dunod | « Annales de géographie »

2006/6 nº 652 | pages 664 à 687

ISSN 0003-4010

ISBN 9782200920968

Article disponible en ligne à l'adresse :

http://www.cairn.info/revue-annales-de-geographie-2006-6-page-664.htm

\section{!Pour citer cet article :}

François Taglioni, «Les petits espaces insulaires face à la variabilité de leur insularité et de leur statut politique », Annales de géographie 2006/6 (n 652), p. 664-687.

DOI 10.3917/ag.652.0664

Distribution électronique Cairn.info pour Armand Colin / Dunod.

(C) Armand Colin / Dunod. Tous droits réservés pour tous pays.

La reproduction ou représentation de cet article, notamment par photocopie, n'est autorisée que dans les limites des conditions générales d'utilisation du site ou, le cas échéant, des conditions générales de la licence souscrite par votre établissement. Toute autre reproduction ou représentation, en tout ou partie, sous quelque forme et de quelque manière que ce soit, est interdite sauf accord préalable et écrit de l'éditeur, en dehors des cas prévus par la législation en vigueur en France. Il est précisé que son stockage dans une base de données est également interdit. 


\title{
Les petits espaces insulaires face à la variabilité de leur insularité et de leur statut politique
}

\author{
Small insular spaces in relation to the variability \\ of their insularity and their political status
}

\section{François Taglioni}

Université d'Artois

Résumé Cette étude interroge les petits espaces insulaires et leurs champs d'étude. II s'agit dans un premier temps de définir les contours de l'objet géographique et ses limites. À défaut de donner une définition absolue de l'île, nous tenterons plutôt de dégager une catégorie que nous appelons les petits espaces insulaires. Ensuite, l'approfondissement de la notion d'insularité permet de remettre certains déterminismes à leur place. Car, si les effets de l'insularité sur le peuplement animal et végétal sont admis depuis longtemps, il en va tout autrement quand on essaie de trouver à l'insularité des influences valides sur les sociétés insulaires. Une esquisse de typologie des insularités sera proposée pour offre quelques pistes et indications sur le niveau de développement et d'intégration des petits espaces insulaires à l'économie-monde. II ne faut néanmoins en aucun cas amplifier les tendances lourdes de cette typologie en des règles ou des lois relatives au poids de l'insularité. La position absolue des îles dans le systèmemonde ne prime pas sur sa position relative par rapport à l'île principale ou à une métropole industrialisée. L'influence des statuts politiques sur les niveaux de développement des petits espaces sera démontrée. La notion de souveraineté partagée sera abordée comme une réponse possible aux évolutions statutaires en milieu insulaire ainsi que pour les populations autochtones qui relèvent de grands États nations.

Abstract This article focusses on small insular spaces. In this this field of study, it has become necessary to first define the nature of the aformentionned geographical object and its limits. As we lack a positive definition of the island, we prefer to emphasize a category called "small insular spaces". Next, a thorough discussion of the notion of insularity should help to set aside some determinisms. Indeed, if the effect of insularity on animal population and vegetation planting has long been accepted, the matter is quite different when one tries to find valid influences on societies. A outline of the various types of insularities will be suggested, and should offer few indications on the level of development and integration of small insular spaces into the world economy. Nevertheless, we should not, and at no cost, transform the greater lines of this typology into rules or laws to measure the weight of insularity. The definite position of islands in the world system does not prevail on its relative position in relation to the main island or to the industrialized home country. The influence of political status will rather be discussed. The notion of shared sovereignty will be tackled as a possible answer to the evolution of status in insular environment, as well as for the local populations who depend on large Nations States. 
Mots-clés Îles, insularité, statut politique, typologie, développement humain, déterminisme.

Key-words Islands, insularity, political status, typology, human development, determinism.

\section{Introduction}

Cet article est une réflexion sur un objet géographique fuyant et lumineux à la fois et sur son champ d'étude. Car, l'île en dépit de son évidence, soulève nombre de questionnements. La première d'entre elle concerne les limites de cet objet. À défaut de donner une définition absolue de l'île, nous tenterons plutôt de dégager une catégorie que nous appelons les petits espaces insulaires. De la Caraïbe, au Pacifique en passant par la Méditerranée et l'océan Indien, ce sont ces espaces sur lesquels la majorité des chercheurs fondent leurs analyses quand ils parlent d'îles. Tenter d'appréhender ces petits espaces insulaires suppose de visiter et de revisiter un certain nombre de concepts et de notions qui façonnent l'objet comme l'insularité, l'" îléité ", l'insularisme, et d'avoir recours à la topologie pour développer les concepts de contiguïté, de connexité, de discontinuité ou encore de métrique, d'enclavement et de périphéricité. Pour cette étude, nous limiterons et axerons principalement notre analyse sur l'approfondissement de la notion d'insularité et de ses liens avec les déterminismes physiques et humains. Face à la variabilité des effets supposés ou avérés de l'insularité, on tentera de dessiner les contours d'une typologie qui ira de l'hypo-insularité à la surinsularité. L'importance des statuts politiques sera enfin abordée pour établir s'il existe une spécificité du statut politique des îles et si celui-ci influe sur le niveau de développement de ces îles.

\section{Essai d'une définition des petits espaces insulaires}

"L'île est profondément paradoxale, ambivalente. Tout se change en son contraire. De là vient qu'il est si difficile d'en saisir l'essence, de la définir, de la fixer. On oscille constamment entre la "banalité de base" et l'indicible. Quand on croit l'avoir bien cernée on la voit qui s'éloigne: certaines îles sont mal amarrées ; ce sont des bateaux qui dérivent et vous échappent »

Anne Meistersheim, 1988, p. 108

Si l'on consulte les dictionnaires et encyclopédies à l'article "île ", on trouve classiquement comme définition « une terre entourée d'eau de toutes parts». L'objet géographique semble donc s'imposer de lui-même avec une grande facilité. Néanmoins, si cette définition est adoptée par tous, pour autant on n'a pas défini les limites qui bornent les îles. Dans les mots de la géographie (Brunet 1993), les auteurs ajoutent qu'une île présente "une taille intermédiaire entre l'îlot et le continent». La belle affaire, quelles sont les limites d'un continent ou d'un îlot? Ultérieurement, Roger Brunet (1997) se risque à 
répondre à la question «quelle est la plus grande île du monde ? ». Il se dégage de sa réflexion, que la Nouvelle-Guinée, et ses 785753 km², symbolise la frontière au-delà de laquelle on ne peut plus parler d'île. Au passage, Roger Brunet donne aussi la limite inférieure de l'île qui serait de $0,5 \mathrm{~km}^{2}$. La NouvelleGuinée donc, on pourrait dire : pourquoi pas ?; mais aussi pourquoi ? Pour Jean-Pierre Doumenge (1984) qui se fonde sur son indice côtier, la NouvelleGuinée est une île continentale au même titre que Madagascar, Bornéo ou Sumatra. Pour la Conférence des Nations unies pour le commerce et le développement (CNUCED, 1983), les États insulaires en développement présentent une population de moins de 400000 habitants et parfois moins de 1 million ainsi qu'une superficie de moins de $700 \mathrm{~km}^{2}$ et parfois supérieure à $4000 \mathrm{~km}^{2}$. Cette définition, fondée sur des critères de viabilité économique, limite grandement le fait insulaire et situe Bahrein ou Ste Lucie aux limites maximales des États insulaires en développement. Christian Huetz de Lemps (1994) dans son étude sur «L'histoire et les îles » s'intéresse lui aux petites îles qu'il définit « avec pour seuil, peut-être, trente mille kilomètres carrés et moins de un million d'habitants». Pour Françoise Péron, "Une île est considérée comme petite quand chaque individu qui y vit a conscience d'habiter un territoire clos par la mer. Une île est considérée comme "grande" lorsque l'ensemble de la société a conscience d'être insulaire, alors que les individus peuvent ignorer ou oublier qu'ils habitent une île " (Péron 1993). Cette définition fait appel aux champs de la représentation, de la vision, du vécu, de l'îléité. Joël Bonnemaison évoque la bonne île qui puiserait ses caractéristiques dans sa bipolarité insulaire : "une "bonne île » est une montagne entourée d'un rivage qui peut servir de port. Grâce à ce "bon rivage", la rupture avec le reste du monde est moins abrupte. Grâce à la montagne, elle dispose d'une profondeur intérieure qui lui permet une certaine diversité »(Bonnemaison 1990, p. 121). Abraham Moles s'amuse, quand à lui, à définir " un cahier des charges pour l'île idéale » (Moles 1982, p. 284).

Il semble bien, que les termes soient absolus (R. Brunet et la plus grande île) ou relatifs (J.-P. Doumenge; la CNUCED, C. Huetz de Lemps, F. Péron, J. Bonnemaison), qu'approcher les îles donne bien des difficultés à quiconque veut les enfermer dans une définition restrictive.

En dépit de l'évidence de l'objet géographique, il n'y a pas de limitation universelle à cet objet. Tout dépend du point de vue où l'on se place : culturel, politique, économique, social. Louis Brigand (2002) reprend dans son ouvrage les définitions des îles par les organisations internationales sur le modèle de la CNUCED. Il est intéressant de noter que pour une même organisation, l'Unesco, les délimitations de l'île vont s'adapter aux finalités des programmes de recherche. Ainsi, pour une étude sur les écosystèmes insulaires, une île, dite "mineure », est "caractérisée par une limite de superficie fixée approximativement à $10000 \mathrm{~km}^{2}$ et moins de 500000 habitants ». Pour une autre étude portant sur l'hydrologie et l'approvisionnement en eau, l'Unesco emploie cette fois comme limite $2000 \mathrm{~km}^{2}$ pour une petite île. Enfin, l'Unesco a créé en 1990 une unité de relations avec 
les petits États membres pour la plupart insulaires et en développement. Ces petits États ont été choisis en fonction des critères suivants : une superficie de moins de $10000 \mathrm{~km}^{2}$, une population de moins d'un million d'habitants et un PNB par habitant d'environ 2000 \$ US.

On observe aussi parfois une évolution des définitions de l'île. C'est le cas notamment de l'ONU, qui suite aux diverses conventions de Genève sur la mer, définit en 1958 l'île comme "une étendue naturelle de terre entourée d'eau qui reste découverte à marée haute ». La Convention des Nations unies sur le droit de la mer sera signée en décembre 1984 à Montego Bay par 117 États souverains. Mais elle entrera en vigueur seulement le 16 novembre 1994 après la $60^{\mathrm{e}}$ ratification. La Convention se donne pour but une appropriation toujours plus forte par les États côtiers des domaines maritimes. La conséquence paradoxale de son fonctionnement est un bénéfice au profit des nations les plus riches (la France, les États-Unis et le Royaume-Uni possèdent les trois plus grandes Zone économique exclusive (ZEE) du monde) alors qu'elle était conçue au départ pour favoriser, sur leur demande, les États les plus pauvres. Les nations développées étaient d'ailleurs hostiles à sa mise en œuvre et ne l'ont signée que tardivement (France, 1996 ; Japon, 1996 ; Pays-Bas, 1996 ; Royaume-Uni, 1997 ; Espagne, 1997 ; Fédération de Russie, 1997) ou ne l'ont pas encore signée (États-Unis). Le corollaire de cette situation est d'avoir multiplié le nombre de revendications et de désaccords entre les États. Pour anticiper ces revendications, il est précisé dans la convention de 1984 que « les rochers qui ne se prêtent pas à une habitation humaine ou à une vie économique propre, n'ont pas de zone économique exclusive ni de plateau continental » (partie VIII, régime des îles, article 21). Dans les faits, ce glissement vers une définition plus restrictive n'empêche pas l'îlot français de Clipperton, qui est «noyé » dans le Pacifique Nord, de jouir d'une ZEE maximale de $431015 \mathrm{~km}^{2}$. Cet îlot de seulement $5 \mathrm{~km}^{2}$ est pourtant inhabité et viole la convention de 1984. Le Mexique le revendique d'ailleurs à la France.

Le point de vue, institutionnel, de l'Union européenne apporte à son tour une définition de l'île. C'est l'Agence européenne de statistiques (Eurostat) qui a tenté de définir les limites statistiques des 25 membres de la Commission des îles. Une île est donc une terre d'au moins $1 \mathrm{~km}^{2}$ de superficie, habitée en permanence par une population statistiquement significative (supérieure à 50 habitants), non reliée au continent par des dispositifs permanents, séparée du continent européen par une étendue d'eau d'au moins $1 \mathrm{~km}^{2}$, ne comprenant pas une des capitales d'un État membre. Ce dernier point sera sans doute à revoir avec l'entrée de Malte et de Chypre dans l'Union européenne à moins de considérer que la République de Malte et Chypre ne sont pas des îles. Par ailleurs, Eubée (3 $655 \mathrm{~km}^{2} ; 220000$ habitants), n'est pas considérée comme une île grecque du fait du pont qui la relie au continent.

On le constate, une grande part d'arbitraire fait loi quand on veut «matérialiser» l'île par des données chiffrées aussi simple qu'un seuil de population ou de superficie. Mais cela n'est-il pas vrai pour tout objet géographique ? Qui est capable de donner les limites absolues entre une colline 
et une montagne par exemple ? La définition des mots de la géographie (Brunet 1993) pour colline est la suivante «Relief de dimension modérée, plus ou moins allongé, se présentant généralement en famille. L’altitude relative est assez faible : 50 à $500 \mathrm{~m}$ environ ». Pour montagne, on peut lire : "Masse de relief d'altitude notable, mais la désignation est relative : la Montagne Noire ne dépasse pas 1200 m, la Montagne de Reims 283 ». À la lecture de ces deux définitions, on voit clairement l'imprécision des limites : La Montagne de Reims pourrait correspondre à une colline. On peut aussi se demander pourquoi on donne comme limite pour la mortalité infantile l'âge d'un an; pourquoi pas 18 mois ou 24 mois ? De la même façon encore, pourquoi borner l'indice de jeunesse par les moins de 15 ans et les plus de 65 ans ?; ne sommes-nous plus jeunes à plus de 15 ans et forcément vieux à plus de 65 ans ?

Il est finalement, on le savait, bien difficile de trouver des éléments scientifiques qui permettraient de définir avec certitude l'île et ses limites. À défaut de s'entendre de façon absolue sur les limites des îles, on peut néanmoins donner une définition de ce que l'on appellera les petits espaces insulaires :

«des terres entourées d'eau de tous côtés, d'un seul tenant, dont la superficie est inférieure à $11000 \mathrm{~km}^{2}$ et la population inférieure à $1,5 \mathrm{mil}$ lion d'habitants ».

La limite de 1,5 million est celle retenue par la Banque mondiale pour définir les petits États, sous-entendu les petites économies. La limite de $11000 \mathrm{~km}^{2}$ permet d'inclure l'archipel des Fidji et sa plus grande île qui est Viti Levu $\left(10531 \mathrm{~km}^{2}\right)$. La limite inférieure de l'île, en deçà de quoi l'on entre dans la catégorie de l'îlot et de l'îlet, sera subordonnée au fait qu'elle soit ou non habitée.

Cette définition apparaîtra, pour certains, bien sujette à caution. Néanmoins, si l'on essaye de rendre les choses plus statistiquement rigoureuses, le résultat sera peu probant. Pour cela, on va s'appuyer sur l'analyse des superficies de 1434 îles. Ces superficies s'étendent de $2 \mathrm{~km}^{2}$ (Farallon de Pajaros, Mariannes du Nord; Herm, îles Anglo-Normandes; Mehetia, archipel de la Société) à $97530 \mathrm{~km}^{2}$ (Mindanao, Philippines). Ce large échantillon ${ }^{1}$ d'îles couvre toutes les mers et tous les océans du monde. Il est extrait d'une base du Programme des Nations unies pour le développement ( UN system-wide earthwatch).

1 Nous avons écarté les extrêmes (extraction de queue) de cet échantillon pour qu'il soit plus homogène. En deçà de $2 \mathrm{~km}^{2}$ on trouve plusieurs centaines d'îles. Au-delà de Mindanao, 97530 km², on trouve 17 États et territoires: I'Islande $\left(101826 \mathrm{~km}^{2}\right)$; Cuba $\left(105805 \mathrm{~km}^{2}\right)$; Luzon - Philippines $\left(109965 \mathrm{~km}^{2}\right)$; North Island - Nouvelle-Zélande $\left(111582 \mathrm{~km}^{2}\right)$; Newfoundland - Canada $\left(115220 \mathrm{~km}^{2}\right)$; Java - Indonésie $\left(138793 \mathrm{~km}^{2}\right)$; South Island - Nouvelle-Zélande (145 $\left.836 \mathrm{~km}^{2}\right)$; Sulawesi - Indonésie (180 $\left.680 \mathrm{~km}^{2}\right)$; Ellesmere - Canada $\left(183964 \mathrm{~km}^{2}\right)$; la Grande-Bretagne Royaume-Uni $\left(209331 \mathrm{~km}^{2}\right)$; Victoria - Canada $\left(220548 \mathrm{~km}^{2}\right)$; Honshu - Japon $\left(225800 \mathrm{~km}^{2}\right)$; Sumatra - Indonésie (443 $\left.065 \mathrm{~km}^{2}\right)$; Baffin - Canada (503 $\left.944 \mathrm{~km}^{2}\right)$; Madagascar $\left(587713 \mathrm{~km}^{2}\right)$; Bornéo - Indonésie, Malaisie, Brunei $(748168$ km²); Nouvelle-Guinée - Indonésie, Papouasie Nouvelle - Guinée (785 753 km²). 


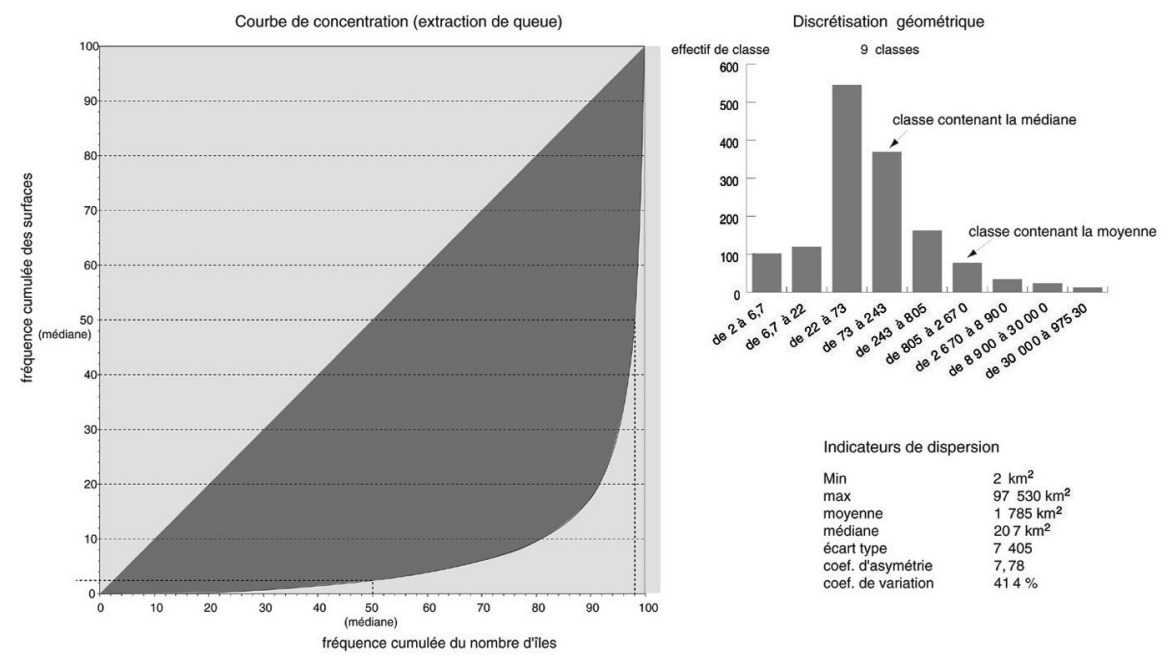

Fig 1 Graphiques de la concentration d'un échantillon mondial d'îles comprises entre $2 \mathrm{~km}^{2}$ et $97530 \mathrm{~km}^{2}$.

A concentration of a world sample of islands from $2 \mathrm{~km}^{2}$ to $97530 \mathrm{~km}^{2}$.

Un traitement statistique ${ }^{2}$ de ces données (Fig. 1) permet de faire les quelques observations suivantes :

- Le coefficient de variation $(414 \%)$ souligne que la série est très étalée

$-50 \%$ des îles, soit 717, ont une superficie inférieure à $207 \mathrm{~km}^{2}$ et surtout $8 \%$ des îles les plus grandes s'accaparent $80 \%$ des surfaces cumulées

- L'écart type est très élevé car les minima et les maxima sont très éloignés

- La discrétisation en 9 classes fait apparaître une distribution uni-modale centrée sur les îles dont la superficie est comprise entre 22 et $75 \mathrm{~km}^{2}$

- Le coefficient d'asymétrie est très supérieur à 0 et met en évidence la surreprésentation numérique des petites îles (superficie comprise entre 2000 et $3000 \mathrm{~km}^{2}$ )

- Les îles d'une superficie supérieure à $11000 \mathrm{~km}^{2}$ sont fort peu nombreuses puisqu'elles représentent moins de $5 \%$ de l'échantillon.

On le voit, tout cela n'est pas hautement significatif, nous continuerons donc, pour le moment, notre analyse avec les limites retenues pour les petits espaces insulaires. De façon empirique encore, les seules exceptions qu'il serait logique de faire concernent la Jamaïque (2,6 millions d'habitants), le Timor-Leste (16 $384 \mathrm{~km}^{2}$ ) et la Nouvelle-Calédonie (16 $648 \mathrm{~km}^{2}$ pour la Grande Terre). Bien que « hors-norme », en termes démographique et de superficie, ces deux États et ce territoire partagent les préoccupations des autres petits espaces insulaires. Cette définition nous conduit à considérer plusieurs milliers de petits

2 Sur l'étude des relations superficies/fréquences et sur la hiérarchisation surfacique voir Depraetere 1991. 
espaces insulaires répartis à la surface du globe. Nous pouvons néanmoins préciser que les petits espaces insulaires sont notamment composés de 33 États indépendants $^{3}$ (archipélagiques pour la grande majorité) (tableau 1) ainsi que de plusieurs dizaines d'outremers associés ou intégrés à des degrés variables à des États continentaux ou archipélagiques (tableau 2). Les petits espaces insulaires se concentrent largement dans le bassin Caraïbe (Petites Antilles), la Méditerranée insulaire, le sud-ouest de l'océan Indien et l'Océanie insulaire.

Tab. 1 Les 33 petits États insulaires dans le monde classés par taille croissante. 33 small island States in the world, by increasing size.

\begin{tabular}{|c|c|c|}
\hline & $\underset{\mathrm{Km}^{2}}{\text { Superficie }}$ & $\begin{array}{c}\text { Population } \\
2003\end{array}$ \\
\hline Nauru & 21 & 12000 \\
\hline Tuvalu & 26 & 11000 \\
\hline Iles Marshall & 181 & 68000 \\
\hline Iles Cook & 237 & 20000 \\
\hline Niue & 259 & 2100 \\
\hline Saint Kitts \& Nevis & 269 & 43000 \\
\hline Maldives & 300 & 320000 \\
\hline Malte & 316 & 400000 \\
\hline Grenade & 345 & 100000 \\
\hline St. Vincent \& Grenadines & 388 & 112000 \\
\hline Barbade & 431 & 268000 \\
\hline Antigua \& Barbuda & 440 & 71000 \\
\hline Seychelles & 455 & 80000 \\
\hline Palau & 488 & 19000 \\
\hline Sainte-Lucie & 616 & 154000 \\
\hline Bahreïn & 665 & 656000 \\
\hline Micronésie & 701 & 133000 \\
\hline Tonga & 747 & 102000 \\
\hline Dominique & 750 & 76000 \\
\hline Kiribati & 811 & 92000 \\
\hline Sao Tomé \& Principe & 1001 & 170000 \\
\hline Comores & 1862 & 615000 \\
\hline
\end{tabular}

3 Sur ces 33 États, 29 sont considérés comme des petits États insulaires en développement. Il s'agit des 33 sans les îles Cook, Chypre, Malte et la Jamaïque (qui n’est pas un petit État insulaire suivant la définition retenue par la Banque mondiale). 
Tab. 1 (suite) Les 33 petits États insulaires dans le monde classés par taille croissante. 33 small island States in the world, by increasing size.

\begin{tabular}{|c|c|c|}
\hline & $\underset{\mathrm{Km}^{2}}{\text { Superficie }}$ & $\begin{array}{c}\text { Population } \\
2003\end{array}$ \\
\hline Maurice & 2045 & 1200000 \\
\hline Samoa & 2935 & 180000 \\
\hline Cap Vert & 4030 & 409000 \\
\hline Trinidad \& Tobago & 5128 & 1285000 \\
\hline Chypre & 9250 & 768000 \\
\hline Jamaïque & 11424 & 2600000 \\
\hline Vanuatu (1) & 12190 & 190000 \\
\hline Bahamas (2) & 13942 & 298000 \\
\hline Fidji (3) & 18333 & 832000 \\
\hline Timor-Leste (4) & 18989 & 800000 \\
\hline Iles Salomon (5) & 28370 & 466000 \\
\hline Total & 137945 & 12552100 \\
\hline
\end{tabular}

Notes: 1. Les plus grandes îles de I'archipel du Vanuatu sont: Esperitu Santo : $3955 \mathrm{~km}^{2}$; Malakula : $2041 \mathrm{~km}^{2}$; Efate : $899 \mathrm{~km}^{2}, 2$. Les plus grandes îles des Bahamas sont: Andros (nord): $3439 \mathrm{~km}^{2}$; Andros (sud) : $1448 \mathrm{~km}^{2}$; Abaco : $1145 \mathrm{~km}^{2}$; Grand Bahama: $1096 \mathrm{~km}^{2}$, 3. Les plus grandes îles des Fidji sont: Viti Levu $10531 \mathrm{~km}^{2}$; Vanua Levu : $5587 \mathrm{~km}^{2}$, 4. La plus grande île est le Timor-Leste : $16384 \mathrm{~km}^{2}$ (2 $461 \mathrm{~km}^{2}$ pour l'enclave de Occuse et $144 \mathrm{~km}^{2}$ pour l'île de Jaco), 5 . Les plus grandes îles des Salomon sont : Guadalganal : $5354 \mathrm{~km}^{2}$; Malaita : $3836 \mathrm{~km}^{2}$; Choiseul : $2970 \mathrm{~km}^{2}$. Les îles en caractères gras sont des îles-États, les autres sont des États multi-insulaires

Tab. 2 Quelques petits territoires insulaires (36) classés par taille croissante et métropoles. Some small island territories (36), by increasing size and by metropole.

\begin{tabular}{|l|c|c|}
\hline & $\begin{array}{c}\text { Superficie } \\
\mathbf{K m}^{\mathbf{2}}\end{array}$ & $\begin{array}{c}\text { Population } \\
\mathbf{2 0 0 3}\end{array}$ \\
\hline Régions insulaires de I'Espagne & & 792000 \\
\hline Baléares & 5014 & 1630000 \\
\hline Canaries (1) & 7242 & \\
\hline
\end{tabular}

\begin{tabular}{|l|r|r|}
\hline Outre-mers de la France & & \\
\hline Wallis et Futuna & 255 & 15000 \\
\hline Mayotte & 374 & 132000 \\
\hline Martinique & 1100 & 382000 \\
\hline Guadeloupe & 1780 & 422000 \\
\hline Réunion & 2504 & 706000 \\
\hline Polynésie française & 3673 & 226000 \\
\hline Corse & 8680 & 260000 \\
\hline Nouvelle-Calédonie & 18575 & 206000 \\
\hline
\end{tabular}

\begin{tabular}{|l|c|c|}
\hline îles Grecques & & \\
\hline Eubée & 3655 & 220000 \\
\hline Crète & 8331 & 560000 \\
\hline Iles loniennes & 1969 & 200000 \\
\hline
\end{tabular}


Tab. 2 (suite) Quelques petits territoires insulaires (36) classés par taille croissante et métropoles Some small island territories (36), by increasing size and by metropole.

\begin{tabular}{|c|c|c|}
\hline & $\underset{\mathrm{Km}^{2}}{\text { Superficie }}$ & $\begin{array}{c}\text { Population } \\
2003\end{array}$ \\
\hline Nord Egée & 3836 & 190000 \\
\hline Sud Egée & 5286 & 271000 \\
\hline \multicolumn{3}{|c|}{ Régions insulaires du Portugal } \\
\hline Madère & 796 & 260000 \\
\hline Açores & 2333 & 245000 \\
\hline \multicolumn{3}{|l|}{ Outre-mer de la Tunisie } \\
\hline Djerba & 592 & 100000 \\
\hline \multicolumn{3}{|c|}{ Outre-mers du Royaume-Uni } \\
\hline Pitcairn & 39 & 47 \\
\hline Anguilla & 91 & 13000 \\
\hline Montserrat & 103 & 4800 \\
\hline Iles Vierges britanniques & 151 & 20000 \\
\hline Iles Cayman & 264 & 38000 \\
\hline Turks \&Caïcos & 417 & 25000 \\
\hline
\end{tabular}

\begin{tabular}{|l|r|r|}
\hline Antilles néerlandaises et Aruba & & \\
\hline Saba & 13 & 1350 \\
\hline Statia & 21 & 2300 \\
\hline St Maarten & 34 & 30600 \\
\hline Curaçao & 444 & 130700 \\
\hline Aruba & 193 & 71000 \\
\hline Bonaire & 288 & 10800 \\
\hline
\end{tabular}

\begin{tabular}{|l|r|r|}
\hline Outre-mers des États-Unis & & \\
\hline Samoa américaines & 200 & 65000 \\
\hline Iles Vierges américaines & 355 & 122000 \\
\hline Mariannes du Nord & 471 & 72000 \\
\hline Guam & 549 & 154000 \\
\hline Hawaï (2) & 16641 & 1200000 \\
\hline
\end{tabular}

Outre-mer de la Nouvelle-Zélande

Tokelau

1500

Total

96281

8779097

Notes : 1. L'île la plus peuplée des Canaries est Gran Canaria avec 716000 habitants, 2. Les plus grandes îles des Hawaï sont : Hawaï $10434 \mathrm{~km}^{2}$; Maui : $1903 \mathrm{~km}^{2}$ et Oahu $1583 \mathrm{~km}^{2}$. Les îles en caractères gras sont des îles-territoires, les autres sont des territoires multi-insulaires 


\section{Insularité et déterminisme}

Pour Françoise Péron (1993), «la spécificité insulaire ne réside pas dans un ou plusieurs caractères qui seraient absolument propres aux îles, mais dans les interactions multiples de données hétérogènes ». L'auteur introduit ainsi la notion de système qui est effectivement fondamentale pour appréhender l'insularité. Néanmoins, l'idée du système suppose que l'insularité est d'autant plus variable que les facteurs seront multiples ou peu nombreux et leurs interactions fortes ou faibles. Toute la difficulté réside donc dans la mise en perspective des facteurs qui définissent l'insularité et des liens qui les unissent. Pour Roger Brunet "l'insularité relève d'un souci d'appréciation objective, externe, empirique, éventuellement fondée sur la mesure » (Brunet 1993, p. 281). Cependant, on sait pour deux des facteurs qui bâtissent l'insularité, la petite taille et l'isolement, combien il est difficile d'en faire une approche quantitative qui soit satisfaisante.

Le flou épistémologique qui entoure l'insularité et l'île n'est pas nouveau et, à travers la littérature (citée par Tissier 1984) de "nos anciens ", on peut lire par ordre chronologique :

"Il est donc impossible de donner de l'insularité une définition simple puisqu'une définition devrait concilier dans une seule formule des caractères généraux contradictoires; il faut étudier les aspects divers de l'existence humaine dans les îles et ne pas prétendre faire de cette diversité une unité illusoire»(Vallaux 1908, p. 110).

"En d'autres termes y a-t-il, peut-il y avoir pour l'anthropogéographie et à sa suite pour l'histoire, une catégorie étiquetée "îles" et valable quelles que soient les circonstances» (Febvre 1922, 227).

"Ici encore on chercherait une nécessité, une loi des îles pesant sur des hommes sur les sociétés humaines; on ne trouverait que variété et diversité » (Febvre 1922, 227).

Et aussi, J. Blache d'ajouter "Y-a-t-il une géographie des îles qui soit autre chose qu'une énumération, un classement commode?» (Blache 1948 , p. 5).

Comme le dit très bien J.-L. Tissier, on est « saisi par le vertige monographique que traduit fort bien un ouvrage comme celui d'Aubert de la Rüe » (Tissier 1984, p. 65).

Peut-être, pourrait-on distinguer l'insularité en fonction de ses effets sur la faune et la flore, d'une part, et sur les sociétés humaines, d'autre part. De nombreux auteurs, et notamment des naturalistes ${ }^{4}$, ont démontré des spécificités physiques du milieu insulaire en fonction de la taille et de l'éloi-

4 «Le problème de l'insularité n'est pas une question de pure verbosité pour les naturalistes " disait J. Blache (Blache 1948, p. 7). Cette phrase n'est pas sans nous ramener à notre verbosité de géographe humain... 
gnement des îles du continent. Certains indices, comme celui d'endémisme de F. Doumenge, permettent de préciser le degré d'isolement d'une île en fonction de l'importance de l'endémisme du peuplement végétal et animal. F. Doumenge, comme d'autres avant lui (cf. notamment Wallace, 1890), précise que "la répartition de toutes les espèces terrestres est directement tributaire de l'éloignement. L'accroissement des distances entraîne l'abaissement rapide du nombre d'espèces présentes dans le peuplement. Cet appauvrissement est de règle des bordures continentales vers le centre de bassins océaniques» (Doumenge 1985, p. 303). Louis Brigand ajoute que "le peuplement animal et végétal des îles s'apparente à une course d'obstacles: la chance de peuplement est d'autant plus faible que l'île est plus éloignée du lieu d'origine des espèces immigrantes. Le taux d'immigration augmente avec la surface de l'île. Il diminue avec la distance au point de départ des espèces colonisatrices et au fur et à mesure que le nombre d'espèces installées s'accroît, du fait des phénomènes de prédation et de compétition entre espèces» (Brigand 2002, p. 25). La cause est donc entendue, il est «des caractères physiques spécifiques au milieu insulaire : morphologie littorale, effet du relief multipliant les zones microclimatiques, variété des paysages sur une superfice réduite, endémisme ${ }^{5}$ biologique, fragilité des écosystèmes » (Benjamin; Godard 1999, p. 56). De ce point de vue, la classification génétique des îles, proposée par von Richthofen au $\mathrm{XIX}^{\mathrm{e}}$ siècle, est précieuse car la taxonomie proposée permet de comprendre les dynamiques naturelles et certaines activités humaines ${ }^{6}$ qui se déroulent sur les îles.

Les quelques considérations qui précèdent sur les effets de l'insularité sur le peuplement animal et végétal sont admises depuis longtemps. Il en va tout autrement quand on essaie de trouver à l'insularité des influences valides sur les sociétés insulaires. Ces éventuelles particularités humaines ou économiques supposeraient que l'insularité soit un concept géographique qui influerait sur l'homme au point que l'on pourrait dire que c'est l'île qui fait l'homme. Cela est bien entendu impensable à moins de replonger tête baissée dans un déterminisme " originel » dont les géographes ont eu bien du mal à se débarrasser ses dernières décennies (cf. Thumerelle 2001). On peut néanmoins postuler que les insulaires sont davantage déterministes que les continentaux ou tout au moins qu'ils font le jeu de croire à l'influence absolue de l'insularité sur leurs conditions de développement. C'est peut-être l'impression qu'ils donnent quand ils s'adonnent à l'excès à l'insularisme.

$\mathrm{Si}$ tout déterminisme est à proscrire, en revanche, envisager l'insularité comme « la relation dynamique qui s'est construite entre un espace insulaire

5 Sur l'endémisme insulaire voir aussi le bel ouvrage illustré du Muséum d'histoire naturelle (Vigne 1997).

6 J. -Ch. Gay a par exemple montré l'importance du rôle du milieu physique sur la mise en tourisme des îles tropicales (Gay 2000). 
et la société qui y vit» (Pelletier 1997a) semble un juste compromis entre un absolutisme déterministe et réducteur, et une négation qui consisterait à dire qu'une île est parfaitement similaire à un autre objet géographique. On peut donc affirmer que l'insularité ne génère pas systématiquement de problématique particulière. Ceci ne veut pas dire que l'on réfute l'enclavement plus ou moins prononcé des îles ou encore leurs spécificités physiques dues à leur taille et à l'isolement. Non, mais ces données ne sont jamais absolues et n'engendrent pas un mal développement qui placerait a priori les îles en position d'isolat ou de marge du système-monde.

On peut enfin, envisager l'insularité en lui apportant une forte charge symbolique pour en faire un terme générique. C'est celui qui fait dire que le monde est un archipel ou que la planète est une île. Déjà, au premier siècle de notre ère, le géographe Strabon parle du monde comme une île. «Que le monde habité soit une île, c'est d'abord l'expérience sensible qui nous force à l'admettre. De tous côtés, en quelque direction qu'il ait été possible d'atteindre les confins de la terre qui nous porte, l'on rencontre la mer, que précisément nous nommons océan : là où il n'est pas donné aux sens de nous le faire admettre, le raisonnement le démontre » (Strabon, cité par Létoublon, 1996, p. 10). Pour Joël Bonnemaison (1997), le monde "peut-être regardé non pas comme un seul espace, mais comme un archipel ». C'est d'ailleurs ce qui fait dire à R. Knafou (in Péron 1996, p. 40) que "l'insularité tend à devenir un facteur faible d'expression d'une différence ». Pour sa part, Hérodote, au ve siècle avant J.-C., envisageait les cinq oasis du désert libyen (Baharia, Dakkhla, Farafra, Kharga, Siwa) comme un archipel des bienheureux. Ostap Guerchenko, géologue à la retraite, l'exprime à sa manière en parlant de la grande ville minière de Norilsk (216 000 habitants) à $400 \mathrm{~km}$ au nord du cercle polaire. " À l'époque soviétique, Norilsk était une ville fermée. Ses habitants étaient arrivés tout jeunes et avaient grandi ici ensemble. Ils étaient devenus tous pareils, comme coulés dans un même moule. Et ils ne voulaient pas des nouveaux venus. C'était comme sur une île» (Libération, jeudi 2 janvier 2003).

Finalement, il semble que l'insularité se mesure plus nettement entre l'île principale et ses îles-satellites qu'entre l'île principale et le continent. Généralement, l'île principale, ou l'île-État dans le cas de la mono-insularité, est parfaitement connectée aux réseaux internationaux de transports aériens, maritimes et d'information. Elle ne souffre donc que faiblement de l'insularité. En revanche, pour les îles secondaires, l'île principale est un cordon ombilical généralement incontournable. À travers plusieurs thèmes, J. - Ch. Gay (1999, p. 81-83) montre bien l'impact des contraintes insulaires en Polynésie française pour les îles secondaires qu'ils qualifient de périphéries de périphéries. "Papeete, toutes choses restant égales par ailleurs, joue le rôle de Paris pour la Polynésie »(Benjamin, Godard 1999). On pourrait encore graduer, et c'est fondamental, les effets de l'insularité par l'intégration plus ou moins avancée de l'île principale à une métropole des pays du 
Nord. Si l'on reste dans le Pacifique insulaire, la situation des habitants de l'archipel des Marquises est plus enviable que celle des habitants des îles Santa Cruz. Le réseau en ligne, qui va en effet de Paris aux Marquises en passant par Tahiti, est bien plus porteur de développement, même pour les îles en fin de chaîne, que celui qui part de Guadalcanal à destinations des Santa Cruz. Les relations distance-temps et distance-coût, primordiales pour envisager l'éloignement, se voient ainsi complétées par une relation distance-statut politique sur lequel nous reviendrons dans la dernière partie.

\section{De l'hypo-insularité à la surinsularité : essai de typologie}

Nous allons essayer d'établir une typologie des insularités. On retiendra pour critères de classification les statuts institutionnels, l'architecture géographique des îles et le niveau de développement en fonction de l'Indice de développement humain (IDH) définit par le Programme des Nations unies pour le développement (PNUD). Il est vrai que cet indice ne reflète pas les disparités régionales de développement. Dans le cas des États et territoires multi-insulaires on perd donc de la finesse dans l'analyse. Ceci est vrai aussi pour les pays continentaux puisqu'on ne descend au niveau de l'IDH des régions. C'est effectivement une des limites de l'IDH mais en attendant des données d'IDH à l'échelle des régions, cet indice est celui qui permet d'appréhender au mieux le développement humain. Pour les territoires dépendant d'une métropole, le niveau de développement se fonde sur des sources variées et des observations de terrain de l'auteur (notamment pour les Petites Antilles, la Méditerranée, le Sud-ouest de l'océan Indien et la Mélanésie). Pour les statuts, on distinguera ainsi les États indépendants, des territoires sous tutelle d'une métropole. Pour l'architecture géographique (cf. Taglioni 2005), on considérera des entités politiques qui sont soit mono-insulaires, soit multi-insulaires avec généralement une île principale et des îles secondaires. Enfin, la classification en trois catégories (développement humain élevé, moyen et faible) de l'IDH, donne une approche correcte du développement. Après le croisement entre ces critères, on dégage une typologie à trois catégories et sept types possibles de situation (fig. 2 et tableau 3). On passera ainsi de l'hypo-insularité (Nicolas 2001), qui serait la continentalisation du phénomène insulaire sous l'impulsion de l'intégration et de l'assimilation d'un territoire insulaire à une métropole continentale, à la surinsularité ${ }^{7}$ (Pelletier 1997b), que l'on pourrait qualifier de double insularité.

Cette typologie nous offre quelques pistes et indications sur le niveau de développement et d'intégration des petits espaces insulaires à l'économiemonde. Les îles qui se trouvent dans la catégorie de l'hypo-insularité sem-

7 L'auteur nous précise: "Les petites îles qui se trouvent en périphérie du bloc central japonais, les ritô, sont caractérisées par ce que j'appelle la "surinsularité". Le rapport entre Japon et continent s'y dédouble par un rapport entre île(s) éloignée(s) et îles centra le(s) (Hondo) ». Pelletier 1997b, p. 134. 
blent mieux intégrées aux rouages de l'économie mondiale que les autres. Leur haut niveau de développement ou leur forte intégration politique ou économique à une métropole industrielle leur donnent les caractéristiques des autres États et territoires du monde développé. Dans ce cas, la discontinuité territoriale est largement compensée par une très bonne accessibilité maritime et aérienne et une parfaite connexité avec le reste de l'économiemonde ou avec une métropole industrialisée qui en fait partie.

En revanche, les îles qui entrent dans la catégorie de la surinsularité sont généralement en retrait des flux d'échanges de biens et de personnes, des réseaux de transports et des routes maritimes et aériennes qui structurent l'espace mondial. D'une façon générale, ces îles, membres secondaires d'un archipel, n'ont qu'une faible marge de manœuvre en matière d'initiative politique, économique ou sociale ce qui les place dans une situation périphérique de l'économie-monde. Leur accessibilité est parfois réduite à un aéroport régional ou à un port, leur connexité se limite aux relations qu'elle entretient avec l'île principale laquelle est elle-même dans une situation de développement précaire.

Pour les îles "simplement insulaires ", tout va dépendre de leur environnement économique, politique et régional. Il semble par exemple que "l'insularité » des îles des Petites Antilles soit moins contraignante que celle de l'Océanie.

Cette typologie n'a qu'une valeur indicative. Il ne faut en aucun cas amplifier les tendances lourdes de cette typologie en des règles ou des lois relatives au poids de l'insularité. La position absolue des îles dans le systèmemonde ne prime pas sur sa position relative par rapport à l'île principale ou à une métropole industrialisée.

\section{Développement et statut politique des petits espaces insulaires}

En prenant en considération la précédente définition des petits espaces insulaires, on ne préjuge pas de leur statut politique. Celui-ci peut aller de la pleine souveraineté reconnue à la dépendance institutionnelle. Dans le premier cas, on parlera alors de micro-État et dans le deuxième de microterritoire. Il existe par ailleurs une troisième catégorie plus discutable qui est celle des micronations. Une des questions est de savoir s'il existe une spécificité du statut politique des îles et si celui-ci influe sur le niveau de développement de ces îles. La première question trouve sa réponse dans la diversité unique des statuts politiques en milieu insulaire dans la mesure où aujourd'hui les confettis d'empires insulaires sont les seuls à ne pas avoir encore accédé à l'indépendance. Ils n'en veulent d'ailleurs pas forcément et la souveraineté partagée est peut-être une réponse adaptée à leur évolution statutaire. Nous allons en faire état maintenant en traitant en parallèle de la relation statut politique/niveau de développement. 


\section{HYPO-INSULARITÉ}

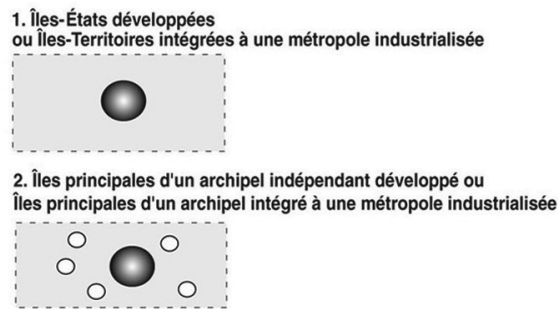

II. INSULARITÉ

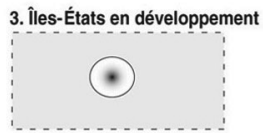

4. îles principales d'un archipel indépendant en développement

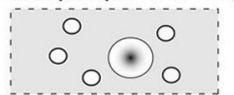

5. îles secondaires d'un archipel intégré à une métropole industrialisée

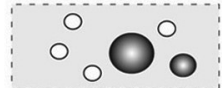

III. SURINSULARITÉ

6. Îles secondaires d'un archipel indépendant en développement

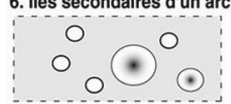

7. Cas particuliers d'îles non côtières sans port ou aéroport

\section{Exemples type}

Bahreïn, Barbade, Chypre

Guam ; La Martinique ; La Réunion

\section{Malte; New Providence}

La Guadeloupe ; Tenerife ; Tahïiti, Oahu

Ste Lucie ; Dominique ; Viti Levu

Trinidad ; St Kitts ; Maurice

St Barthélémy

Barbuda ; Rodrigues ; Esperitu Santo

Certaines îles éloignées de la Japonésie ou de l'Océanie insulaire
Île principale développée ou intégrée à une métropole développée

Île principale en développement

Limite politique
Île secondaire intégrée à une métropole développée

○) île secondaire en développement

Autre île secondaire

F. TAGLIONI

Fig 2 Schéma d'une typologie des insularités dans les petits espaces insulaires. Diagram of a typology of insularities and of small insular spaces.

\subsection{Les micro-États : une lente reconnaissance}

Depuis la déclaration de David Wainhouse selon lequel l'indépendance est "une solution extravagante et inadéquate pour les petits territoires » (Wainhouse 1964, p. 133 cité par Blair 1968, p. 6) le chemin parcouru a été long. Entre 1960 et 2000 , c'est la totalité des petits États insulaires qui a été admise à l'ONU. Seules les îles Cook et Niue, qui possèdent des gouvernements autonomes en libre association avec la Nouvelle-Zélande, ne 
Tab. 3 Tableau d'une typologie des insularités dans les petits espaces insulaires.

Diagram of a typology of insularities in small island spaces.

\begin{tabular}{|c|c|}
\hline \multirow[t]{2}{*}{ Hypo-insularité } & $\begin{array}{l}\text { Îles-États développées où } \\
\text { Îles-Territoires intégrées à une métropole industrialisée (1) }\end{array}$ \\
\hline & $\begin{array}{l}\text { Îles principales d'un archipel indépendant développé où } \\
\text { îles principales d'un archipel intégré à une métropole industrialisée (2) }\end{array}$ \\
\hline \multirow[t]{2}{*}{ Exemples } & 1 : Bahreïn ; Barbade ; Chypre ; Guam ; La Martinique ; La Réunion \\
\hline & 2: Malte ; New Providence ; La Guadeloupe ; Tenerife ; Tahiti ; Ohau \\
\hline \multirow[t]{3}{*}{ Insularité } & Îles-États en développement (3) \\
\hline & Îles principales d'un archipel indépendant en développement (4) \\
\hline & Îles secondaires d'un archipel intégré à une métrople industrialisée (5) \\
\hline \multirow[t]{3}{*}{ Exemples } & 3: Ste Lucie ; Dominique ; Viti Levu \\
\hline & 4 : Trinidad; St Kitts ; Maurice \\
\hline & 5 : St Barthélémy \\
\hline \multirow[t]{2}{*}{ Surinsularité } & Îles secondaires d'un archipel indépendant en développement (6) \\
\hline & Cas particuliers d'îles non côtières sans port ou aéroport (7) \\
\hline \multirow[t]{2}{*}{ Exemples } & 6: Barbuda; Anjouan; Rodrigues; Esperitu Santo \\
\hline & 7: Certaines « îles éloignées» de la Japonésie ou de l'Océanie insulaire \\
\hline
\end{tabular}

sont pas reconnus comme pleinement indépendants et ne peuvent donc pas siéger à l'ONU. Ces admissions sont la reconnaissance pleine et sans restrictions de l'existence, en qualité d'État à part entière, d'entité géographique ayant des seuils de population de l'ordre de 11000 habitants pour Tuvalu et de superficie de $21 \mathrm{~km}^{2}$ pour Nauru. François Doumenge montre que cette prolifération de micro-États insulaires a posé dans les années 1960 la question de leur viabilité économique et politique (Doumenge 1985). Finalement, les années ont passé et de nombreux groupes d'experts d'organisations internationales ont fourni des rapports entre 1969 et $1984^{8}$ pour arriver à une conclusion qui s'est imposée d'elle-même : les microÉtats existent et ils trouvent leur place dans le concert des nations.

François Doumenge faisait remarquer en 1985 que le volume économique moyen des admis accusait une forte tendance à la baisse entre 19641973 et 1974-1983. On confirme cette baisse avec la vague d'admission, de 1991 à 2004, d'États qui possèdent des économies ayant des PNB moyens de 0,1 milliard de dollars américains. Cette ultime vague ${ }^{9}$, qui consacre

8 On en trouvera une liste non exhaustive dans I'article de F. Doumenge 1985, p. 326-327.

9 Elle concerne les îles Marshall (1991), la Micronésie (1991), Palau (1994), Nauru (1999), Kiribati (1999), les Tonga (1999) et le Timor oriental (2002). 
les micro-États insulaires, concerne uniquement l'Océanie insulaire et les presque ${ }^{10}$ derniers confettis d'empire de la région. Il est à noter que le Timor oriental est entré aux Nations Unies dès son indépendance en mai 2002. Les efforts financiers et diplomatiques des Nations unies, pour parvenir au règlement du conflit avec l'Indonésie et amorcer la reconstruction du pays, montrent bien que les petites nations sont des nations comme les autres au regard du droit international.

Fabrice O'Driscoll apporte une distinction qui fonde une nouvelle catégorie de micro-États, ceux dont la souveraineté internationale n'a pas été reconnue de façon bilatérale ou multilatérale par d'autres acteurs de l'ordre public mondial. «Le plus souvent, le micro-État reconnu est membre des Nations unies ${ }^{11}$. Le micro-État non reconnu est, a contrario, le micro-État qui, tout en possédant l'ensemble des caractéristiques du micro-État reconnu, n'a pas (encore) obtenu le même statut officialisant sa position. Précisons que la reconnaissance ou la non-reconnaissance d'un micro-État (et il en va de même pour les États) est sans rapport avec sa validité : la reconnaissance d'un micro-État (ou d'un État) relève de l'opportunité politique alors que sa validité repose sur la réunion de critères objectifs. Hutt River ${ }^{12}$ ou Taiwan ne sont pas officiellement reconnus, mais cela ne les empêche pas d'exister ! »(O'Driscoll 2000).

À l'intérieur de ce groupe des micro-États, la relation superficie/PNB/ h ou population $/ \mathrm{PNB} / \mathrm{h}$ ne se vérifie pas. Les situations sont extrêmement variées et les corrélations ne sont pas de mise. On va voir que le statut politique en revanche, est un déterminant du niveau de développement.

\subsection{Les microterritoires : un paradoxe de l'histoire}

Nous employons ici l'expression de microterritoires insulaires pour désigner des espaces insulaires qui dépendent, à divers degrés, d'une métropole souvent fort éloignée. On remarquera du reste qu'il n'existe pratiquement aucun territoire continental qui appartienne à une métropole sans y être territorialement rattaché. Ce constat renforce l'idée que la mer, qui délimite par son action tomogène des frontières naturelles, est propice à l'existence des microterritoires. Ces microterritoires insulaires sont en grande majorité les expressions ultimes des empires coloniaux européens, des fins de réseaux. Ces associats sont aujourd'hui dispersés sur tous les océans et mers du monde. Les mères patries sont européennes (Danemark, Espagne, France, Pays-Bas, Portugal, Royaume-Uni, Suède), australiennes, chiliennes, équatoriennes, états-uniennes ou néo-zélandaises. Ces microterritoires ont

10 Il ne reste plus que la Nouvelle-Calédonie, Wallis et Futuna, la Polynésie française, Pitcairn, Tokelau, Guam, les Mariannes du Nord et les Samoa américaines.

11 À ce jour, seul le Saint-Siège est un État reconnu non membre de l'ONU.

12 Le cas du micro-État de Hutt River en Australie est bien décrit par Fabrice O'Driscoll (O'Driscoll 2000, p. 94-97). Voir aussi le site Internet : http://www.geocities.com/CapitolHill/5829/consulat.html. 
des statuts fort différents au regard des droits nationaux, communautaires ou internationaux.

En voici quelques exemples pris en France, au Royaume-Uni, aux PaysBas, aux États-Unis et en Nouvelle-Zélande. Pour les seuls outremers français, on dénombre 6 groupes de statut dans le droit national. Il s'agit de l'intégration la plus forte à la moins forte des Départements d'outre-mer (les 4 DOM), de la collectivité départementale de Mayotte, de la collectivité territoriale de Saint-Pierre-et-Miquelon, du territoire d'outre-mer de Wallis-et-Futuna, du Pays d'outre-mer de la Polynésie française et de la collectivité nommée de Nouvelle-Calédonie. Pour les outremers du RoyaumeUni, c'est le statut de colonie qui est toujours en place. Les colonies sont néanmoins largement autonomes et elles ont chacune un conseil exécutif et un parlement législatif (chambre législative, et conseil législatif élu au suffrage direct). Ce statut est à rapprocher de celui de l'île de Tokelau qui est administrée par la Nouvelle-Zélande. Les Antilles néerlandaises forment une région autonome du Royaume tripartite des Pays-Bas. Aruba est la deuxième composante du Royaume et les Pays-Bas la troisième. Pour Aruba et les Antilles néerlandaises, il n'y a donc plus véritablement de rapport de territoires dépendants à une métropole puisque celle-ci est une des nations composant l'État. Les territoires américains sont membres du Commonwealth des États-Unis. L'exécutif est aux mains d'un gouverneur élu au suffrage universel et le législatif est assuré par un sénat, élu, lui aussi, au suffrage universel.

Sur le plan international, les microterritoires, que l'on vient de citer, n'ont pas de statut particulier. Ils souffrent du reste, à cause de leur assimilation à une métropole, d'un manque de données socio-économiques qui les caractériseraient plus finement. Ils entrent bien trop souvent dans un cadre statistique national rigide qui ne les différencie pas du reste du territoire métropolitain. Il semble que les États aient tendance à faire des agrégats des entités géographiques. C'est le cas manifeste des DOM français.

Au regard du droit communautaire en revanche, les microterritoires de l'Union européenne, hors territoire douanier, jouissent d'une reconnaissance particulière en qualité de Pays et territoire d'outre-mer (PTOM).

La grande diversité des statuts dont il vient d'être fait état, ne masque pas la question d'une évolution institutionnelle possible des microterritoires. Les réponses seront à géométrie variable en fonction de la situation de départ. Pour les microterritoires solidement ancrés dans une métropole le statu quo est de mise bien qu'un approfondissement de leurs pouvoirs locaux soit envisageable. C'est typiquement la situation dans les départements français d'outre-mer par exemple. Les habitants des Antilles françaises se sont ainsi prononcés, le 7 décembre 2003, par référendum sur un vaste, quoique flou, projet de réforme institutionnelle. Les électeurs de la Guadeloupe et de la Martinique ont finalement repoussé ce projet. En revanche, Saint-Martin et Saint-Barthélemy ont choisi d'opter pour un nouveau statut de collectivité d'outre-mer qui leur permet de préserver leurs acquis fiscaux et les libère de la tutelle de la Guadeloupe dont 
elles étaient des communes. La liste des outremers français ${ }^{13}$ s'allonge donc aujourd'hui de deux nouvelles entités en devenir institutionnel. Dans l'océan Indien, la situation est bien différente puisque les hommes politiques Réunionnais défendent bec et ongles le statu quo et ne veulent en aucun cas que l'État puisse mentionner dans la constitution une possible évolution statutaire de ses DOM. La crainte des Réunionnais est de l'ordre de la mathématique. Le transfert de l'État vers les collectivités locales peut-il s'envisager comme une opération à somme nulle ? Autrement dit, ces collectivités gagneront-elles ce que l'État perdra? La réponse est pour le moins incertaine et certainement à géométrie variable suivant les collectivités institutionnelles et géographiques considérées. La deuxième option est celle d'un très large transfert des compétences de l'État vers les microterritoires. C'est aujourd'hui le cas de la Polynésie française, de la Nouvelle-Calédonie ou encore des Antilles néerlandaises et d'Aruba. Pour ces territoires, il semble que seule la Nouvelle-Calédonie soit engagée vers une possible indépendance totale qui dépendra du vote de la population à partir de 2014 conformément aux accords de Nouméa de 1998. Les autres territoires devraient en revanche connaître eux aussi un statu quo dans les années à venir. Enfin, les territoires britanniques et américains, peut-être les plus à même à exprimer leurs souhaits de recouvrir l'indépendance, ne manifestent nullement, à l'exception de Guam, leur volonté de décolonisation alors même que leur métropole de tutelle les y encourage. C'est le paradoxe de l'histoire de peuples qui optent pour le maintien de leur statut en refusant l'indépendance qui leur est offerte; les temps changent... Le comité spécial de l'ONU, chargé d'étudier la situation en ce qui concerne l'application de la Déclaration sur l'octroi de l'indépendance aux pays et aux peuples coloniaux, continue néanmoins de publier régulièrement des rapports sur la situation des territoires non-autonomes dans le monde en vue de leur indépendance.

On s'étonnera cependant que les entités insulaires reçoivent exclusivement l'attention de ce comité spécial de l'ONU. Pourtant, il existe aussi nombre de territoires continentaux ${ }^{14}$ qui sont en situation d'enclave/exclave et qui revendiquent parfois l'autonomie ou l'indépendance. On pense notamment à l'enclave angolaise de Cabinda qui s'estime indépendante depuis 1975, date de l'indépendance de l'Angola. Un président, António Luis Lopes, et un gouvernement autoproclamé tentent de se faire entendre de par le monde mais le Cabinda reste occupé par l'armée angolaise.

On a constaté précédemment que la relation superficie/PNB/h ou population/PNB/h ne se vérifie pas pour les micro-États. Ce constat reste entier pour les microterritoires qui présentent des PNB globaux et par

13 Pour une mise au point sur statuts les statuts des outre-mers français voir Gay 2005.

14 On peut citer pêle-mêle : Kaliningrad, enclave russe entre Pologne et Lituanie ; Llivia, enclave espagnole dans les Pyrénées françaises ; Occuse enclave du Timor oriental dans le Timor indonésien ; Ceuta et Melilla, enclaves espagnoles au Maroc ; la péninsule de Mussamdam, enclave de I'Oman en périphérie littorale nord des Émirats Arabes Unies; le Sultanat de Brunei séparé en deux par la partie malaise de Bornéo et encore, Gibraltar, l'Alaska et d'autres (sur ce sujet lire : http://home.no.net/enklaver/exclaves.htm). 
habitant d'une grande variabilité sans attaches notables avec la superficie ou la population. En revanche, il est très clair que les microterritoires ont l'indicateur moyen du $\mathrm{PNB} / \mathrm{h}$ largement supérieur à celui des micro-États. Il s'établit dans le premier cas à $9300 \$ /$ h et à $4500 \$ /$ h dans le deuxième. Il faut sans doute voir ici, dans la majorité des cas, l'expression des transferts financiers et de la solidarité de la métropole vers ses territoires associés. Dans d'autres cas, minoritaires, c'est la bonne santé économique qui génère les richesses des microterritoires. On pense notamment aux plus riches et particulièrement à la Nouvelle-Calédonie et ses mines de nickel ou encore aux îles Cayman qui abritent plus de sociétés offshore que d'habitants. Ce tableau assez général est un facteur d'explication des réticences des microterritoires à demander leur indépendance alors que l'économie mondiale est de plus en plus interdépendante et sensible à la conjoncture politique mondiale. Et puis, il est des statuts d'autonomie interne qui sont bien plus attractifs et rassurants qu'une indépendance aux lendemains incertains.

On pense ici particulièrement à celui des îles d'Aland, un petit archipel (fig. 3) à l'entrée du golfe de Botnie qui compte 26000 habitants. Ils se répartissent sur plus de 6000 îles, dont 80 sont habitées, pour une superficie

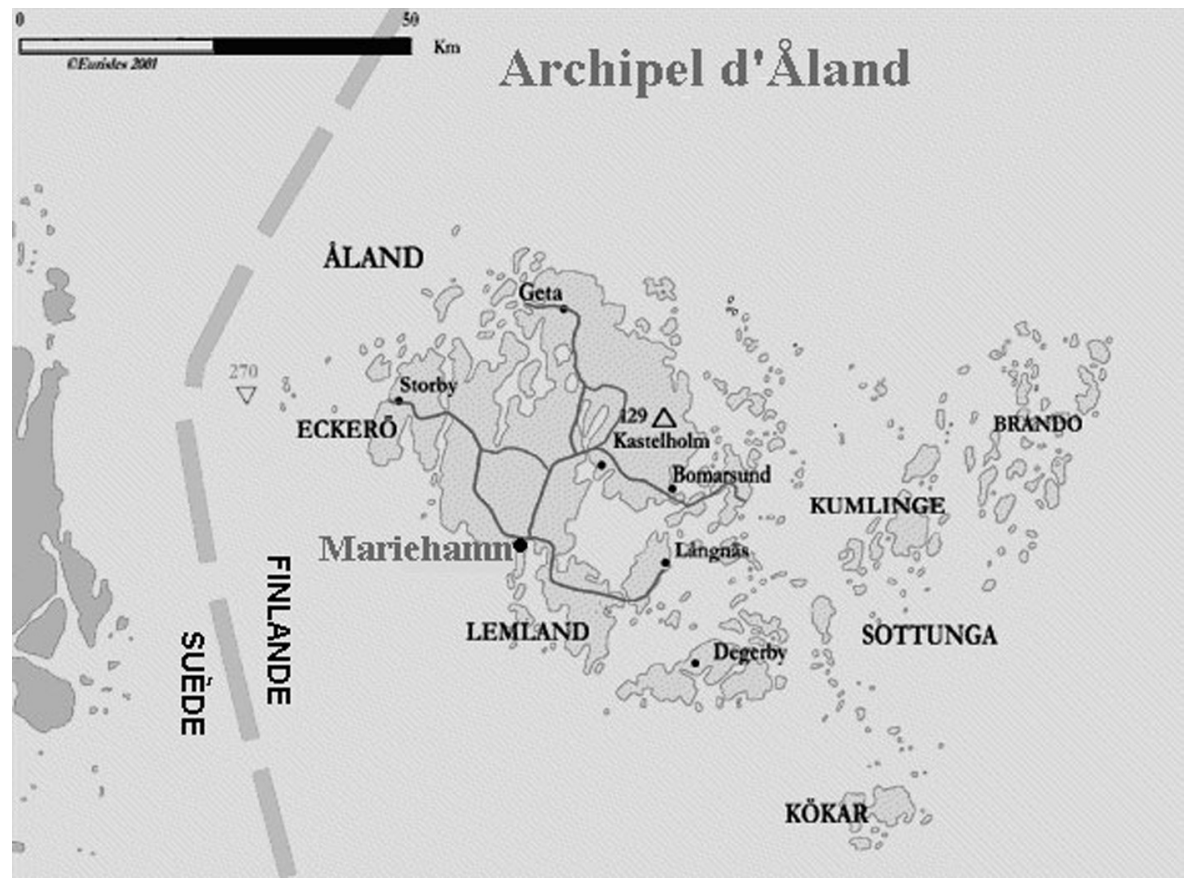

Source : http://www.tlfq.ulaval.ca/axl/europe/alandcarte.htm

Fig 3 L'archipel des Aland, entre Suède et Finlande.

Aland archipelago between Sweden and Finland. 
totale de $1524 \mathrm{~km}^{2}$. L'île principale, Aland (Ahvenanmaa) a une superficie de $720 \mathrm{~km}^{2}$. Elle accueille la capitale, Mariehamn, ainsi que la grande majorité de la population de l'archipel auquel elle a donné son nom. L'archipel forme une des six provinces finlandaises à laquelle il est institutionnellement rattaché depuis l'indépendance de la Finlande en 1917. La population est néanmoins suédophone en grande majorité et une petite minorité parle le finlandais. Après une longue bataille juridique internationale, la loi sur l'autonomie du 28 décembre 1951 consacre les très larges pouvoirs législatifs et administratifs de l'archipel.

Le statut des Aland est assez comparable à celui des îles Féroé (46 000 habitants, $1400 \mathrm{~km}^{2}$ ) qui sont elles aussi largement indépendantes, depuis la création d'un parlement le 23 mars 1948, au sein de la monarchie constitutionnelle du Danemark. Le statut de ces deux archipels pourrait servir de modèle à nombre d'archipels et de régions continentales qui ne se reconnaissent pas complètement dans une métropole et qui revendiquent plus ou moins violemment le droit à la différence jusqu'à mettre parfois en doute les États nations de tutelle. Ce statut, qui débouche sur la notion ambiguë de souveraineté partagée (Agniel, G. ; Faberon J.-Y., 1999), souligne que la souveraineté n'est plus le monopole de l'État. La Nouvelle-Calédonie, et sa citoyenneté néo-calédonienne distincte, en fournit un autre bon exemple. Cette forme hybride, entre souveraineté et dépendance, pourrait servir de modèles pour d'autres microterritoires, s'ils le demandent, en devenir institutionnel comme les îles Cook ou Niue associées à la Nouvelle-Zélande, les îles Vierges américaines, ou encore Anguilla et Montserrat, colonies britanniques. La souveraineté partagée pourrait aussi apporter une réponse aux populations autochtones qui relèvent de grands États nations comme le Brésil, le Canada, l'Afrique du sud ou l'Australie.

\subsection{Les micronations : plus virtuelles que réelles}

L'approfondissement de la notion de micronation est dû à l'Institut français de micropatrologie ${ }^{15}$. C'est une association qui se voue à l'étude des petits pays dans le monde. Son président, Fabrice O’Driscoll, a rédigé en 2000 un ouvrage qui recense et nous éclaire sur plus de 600 micro-États non reconnus et micronations confidentielles. L'auteur entend par micronation "une très petite nation, c'est-à-dire une collectivité humaine organisée, soumise le plus souvent à un gouvernement et à des lois communes mais hors d'un espace donné. La micronation n’évolue pas sur un territoire délimité et certaines d'entre elles récusent d'ailleurs toute revendication» (O’Driscoll 2000). Cette déterritorialisation du politique entraîne une rupture des codes et donne à l'imagination la pleine possibilité de s'exprimer sur un monde que certains souhaitent différent. Les micronations sont, dans la grande majorité des cas, virtuelles et elles doivent leur existence

15 Cette association est dans la continuité de l'International micropatrological society créée en 1973 par le Britannique F. Lehmann. 
propre aux possibilités qu'offre le réseau Internet. Nous citerons pour exemple le Saint Empire de la Réunion (Holy Empire of Reunion) qui est une parodie brésilienne du département français d'outre-mer ${ }^{16}$. On imagine mal, en revanche, un groupe d'étudiants, comme dans le cas du Saint Empire de la Réunion, annexer un pays comme les États-Unis ou la Russie.

Les petits espaces semblent plus permissifs que de vastes ensembles continentaux. On peut aisément les ranger dans la catégorie des «antimonde » de la finance, de la drogue, du sexe, du carcéral et d'expérimentations en tout genre à l'abri des regards. Partant, il apparaît plus facile de se les "approprier", c'est toujours l'effet garanti du "small is beautiful». C'est ce qui explique que les micronations ne s'étendent généralement pas au-delà de quelques $\mathrm{km}^{2}$.

\section{Conclusion}

Au terme de cette étude se pose la question de la spécificité de l'objet géographique, l'île. Mais n'est ce pas un faux problème ? Les urbanistes éprouvent quelques difficultés à définir la ville et pourtant elle existe et ils l'étudient. Il faut donc éviter de trop focaliser sur l'objet, comme le fait A. Moles, 1982, quand il parle d'une science de l'île (nissologie). En fait, la thématique n'est pas l'île mais bien plutôt l'insularité, l'« îléité ", l'insularisme et les concepts afférents d'isolement, de contiguïté, de connexité, de discontinuité, d'enclavement et de "périphéricité ». "Certes, les prérequis de la construction du champ interfèrent sur la construction de l'objet ", mais cela ne doit amener à une "confusion entre l'objet en soi et les méthodes d'appréhension de l'objet en question» (Pelletier 2005). C'est dans ce sens, qu'au-delà d'une définition possible d'une catégorie d'îles que nous désignons par petits espaces insulaires, nous avons essayé d'appréhender l'insularité et sa variabilité ainsi que l'influence possible des statuts politiques sur les niveaux de développement des petits espaces marqués par l'insularité. On ne peut pas, néanmoins, préjuger et généraliser les effets supposés de l'insularité, quelle que soit la forme qu'elle prend.

\footnotetext{
Université d'Artois

BP 665, 62030 Arras

tagli@diplomacy.edu
}

16 Voir le site officiel du Saint Empire de la Réunion : http://www.reuniao.org/ 


\section{Bibliographie}

Agniel, G. ; Faberon J.-Y. (1999). La souveraineté partagée en Nouvelle-Calédonie et en droit comparé. Paris : La Documentation française, 180 p.

Benjamin D. ; Godard, H. (1999). Les outremers français : des espaces en mutation. Paris : Ophrys, $267 \mathrm{p}$.

Blache J. (1948), "Les particularités géographiques des îles ». Bulletin de la société de géographie $d^{\prime}$ Aix-Marseille, $\mathrm{n}^{\circ}$ 44, p. 5-22.

Bernardie, N. ; Taglioni, F. (dir.). Les dynamiques contemporaines des petits espaces insulaires. Paris : Karthala, 443 p.

Blair P. (1968). Le problème des micro-États. Genève : Dotation Carnegie pour la paix internationale, $94 \mathrm{p}$.

Bonnemaison J. (1997). "La sagesse des îles ». In Sanguin, A.-L. (dir.). Vivre dans une île. Paris : I'Harmattan, p. 121-129.

Bonnemaison J. (1990). "L'espace réticulé ». In Tropiques, lieux et liens. Paris : Orstom, p. 500510.

Brigand L. (2002). Les îles du Ponant. Plomelin : Éditions Palantines, 480 p.

Brunet R. (1997). "Quelle est la plus grande île du monde ? ». MappeMonde, n 4, 40-41.

Brunet R., Ferras R., Théry H. (1993). Les mots de la géographie, Paris/Montpellier : La Documentation française/Reclus, $520 \mathrm{p}$.

Action spécifique se rapportant aux besoins et aux problèmes particuliers des pays en développement insulaires (1983). Belgrade : CNUCED VI. TD/279, 33 p.

Depraetere, C. (1990-1991). «Le phénomène insulaire à l'échelle du globe : tailles, hiérarchies et formes des îles océanes ». L'Espace géographique, n², p. 126-134

Doumenge F. (1985). «Les îles et les micro-États insulaires ». Hérodote, n 37-38, 297-327.

Doumenge F. (1984). «Unité et diversité des caractères naturels des îles tropicales ». In Nature et hommes dans les îles tropicales. Bordeaux : CRET, coll. "îles et archipels », n³, p. 9-24.

Febvre L. (1922). La Terre et l'évolution humaine. Paris : La renaissance du livre, 471 p.

Fuligni, B. (1997). L'État, c'est moi. Histoire des monarchies privées, principautés de fantaisie et autres républiques pirates, Paris : Édition de Paris, $238 \mathrm{p}$

Gay J.-Ch. (2005). "Où en est la France d'outre-mer? ». Mappemonde, n 79

Gay J.-Ch. (2000). "La mise en tourisme des îles intertropicales ». Mappemonde, n 58, p. 17-22.

Gay J.-Ch. (1999). Recherches sur les discontinuités spatiales et le tourisme. Paris : Université de Paris VII, Habilitation à diriger des recherches, $228 \mathrm{p}$.

Huetz de Lemps C. (1994). «L'histoire et les îles... ». Hérodote, n 74/75, p. 2-45.

Knafou R. (1996) in Péron F. (dir.). " Les îles en société : fonctions sociales, dimensions subjectives et intégration au système monde », Séminaire de I'URA 904, Brest, 3-5 avril, p. 40.

Létoublon F. (dir.) (1996). Impressions d'îles. Toulouse : Presses universitaires du Mirail, 294 p.

Meistersheim A. (1988). "Insularité, insularisme, iléité, quelques concepts opératoires ». Cahiers de I'institut de développement des îles méditerranéennes, $\mathrm{n}^{\circ}$.1, p. 96-120.

Moles A. (1982). "Nissonologie ou sciences des îles ». L'Espace géographique, n 4, p. 281-289.

Nicolas, T. (2001). « La circulation comme facteur d'intégration nationale et d'hypo-insularité ». Les cahiers d'outre-mer, $\mathrm{n}^{\circ} .216$, p. 397-416.

O'Driscoll F. (2000). Ils ne siègent pas à I'ONU : revue de quelques micro-Etats, micronations et autres entités éphémères. Toulon : Presses du Midi, 287 p.

Pelletier P. (2005). "L'île, un bon objet géographique ». In Bernardie, N. ; Taglioni, F., (dir.), Les dynamiques contemporaines des petits espaces insulaires. Paris : Karthala, p. 7-17.

Pelletier P. (1997a). La Japonésie : géopolitique et géographie historique de la surinsularité au Japon. Paris: CNRS, 391 p. 
Pelletier P. (1997b). "Aspects géopolitiques de la surinsularité japonaise ». In Sanguin, A.-L. (dir.), Vivre dans une île. Une géopolitique des Insularités. Paris : L'Harmattan, p. 131-141.

Péron F. (1993). Des îles et des hommes. Rennes : Édition de la Cité/Ouest-France, 286 p.

Sanguin A.-L. (1981). " "Small is not beautiful" : la fragmentation politique de la Caraïbe ». Cahiers de géographie du Québec, n 66, p. 343-360.

Taglioni F. (2005). «Les revendications séparatistes et autonomistes au sein des États et territoires multi-insulaires. Essai de typologie », Cahiers de géographie du Québec, n 146, p. 5-18, http:/ /www.cgq.ulaval.ca/textes/vol_49/no136/03-Taglioni.pdf

Taglioni F. (2003). Recherches sur les petits espaces insulaires et sur leurs organisations régionales. Paris : Université Paris-IV, mémoire d'habilitation à diriger des recherches, volume II, 218 p. http://www.Taglioni.net/hdr.htm.

Thumerelle P.-J. (dir.) (2001). Explications en géographie. Paris : Sedes, 160 p.

Tissier J.-L. (1984). « Îles, insularité, isolement ». Documents pour l'histoire du vocabulaire scientifique, $\mathrm{n}^{\circ} .3$. Paris : CNRS-GRECO, p. 49-67.

Vallaux C. (1908). Géographie sociale. Paris : O. Doin, 377 p.

Vigne J.-D. (dir.) (1997). Îles, vivre entre ciel et mer. Paris : Nathan/Museum national d'histoire naturelle, $127 \mathrm{p}$.

Wainhouse D. (1964). Remnants of Empire: The UN and the End of Colonialism. New York: Harper \& Row, 225 p.

Wallace A. (1880). Island life, Londres : Macmillan, 526 p. 